\title{
Association between deposit of abdominal adipose tissues and metabolic abnormality in a Chinese population
}

\section{Xuhui Zhang}

Hangzhou Center for Disease Control and Prevention

\section{Qiannan Chen}

Zhejiang Chinese Medical University

Xiaohui Sun

Zhejiang Chinese Medical University

Qiong Wu

Zhejiang University

\section{Zongxue Cheng}

Zhejiang University

\section{Qingguo Lv}

Sichuan University West China Hospital

\section{Jiaqiang Zhou}

Zhejiang University School of Medicine Sir Run Run Shaw Hospital

\section{Yimin Zhu ( $D$ zhuym@zju.edu.cn )}

Zhejiang University https://orcid.org/0000-0001-8409-7636

\section{Research Article}

Keywords: Obesity, Subcutaneous adipose tissue (SAT), Visceral adipose tissue (VAT), body mass index (BMI), metabolic abnormality (MA)

Posted Date: August 30th, 2021

DOl: https://doi.org/10.21203/rs.3.rs-818177/v1

License: (c) (i) This work is licensed under a Creative Commons Attribution 4.0 International License. Read Full License 


\section{Abstract}

\section{Background}

Previous studies indicated that deposit of abdominal adipose tissue associated with the abnormalities of cardiometabolic components. However, the results were inconsistent on the effects of subcutaneous adipose tissue (SAT) and difference between men and women. The aim of this study was to examine the associations of VAT, SAT with metabolic status and the different effects between male and female.

\section{Methods}

1388 eligible subjects were recruited from the baseline investigation of metabolic syndrome investigation in China. Areas of abdominal VAT and SAT were determined by nuclear magnetic resonance imaging (MRI). Total triglycerides (TG), high-density lipoprotein cholesterol (HDL-C) were measured by biochemical auto-analyzer. Metabolic abnormality (MA) was defined more than one of abnormally metabolic components, which were based on the definition of metabolic syndrome (IDF 2005). Multiple logistic regression was used to calculate the odds ratios $(\mathrm{ORs})$ and $95 \%$ confidence intervals $(95 \% \mathrm{Cl})$. The predictive values were assessed with area under the curve (AUC), net reclassification improvement (NRI), and integrated discrimination improvement (IDI), respectively.

\section{Results}

Subjects with MA had higher levels of VAT in both of men and women, and higher levels of SAT in men than those with metabolic normality $(\mathrm{MN})(P<0.05)$. Different associations of SAT in women depended of levels of BMI. Higher levels of VAT were significantly correlated with higher risks for MA ( $p$ for trend $<0.05$ ). Comparing with the subjects of the first quartile (Q1) of VAT, OR in fourth quartile (Q4) was 6.537 $(95 \% \mathrm{Cl}=3.394-12.591)$ in men and $3.364(95 \% \mathrm{Cl}=1.898-5.962)$ in women. However, no significance was found in SAT. In men, VAT improved the predictive value of MA with the AUC of 0.727 (95\% Cl= 0.687 $0.767)$, and $\mathrm{NRI}$ of $0.139(95 \% \mathrm{Cl}=0.070-0.208)$ and $0.106(95 \% \mathrm{Cl}=0.038-0.173)$, IDI of $0.074(95 \% \mathrm{Cl}=$ 0.053-0.095) and $0.046(95 \% \mathrm{Cl}=0.026-0.066)$ comparing with $\mathrm{BMI}$ and $\mathrm{WC}$, respectively. Similar results were found in women.

\section{Conclusions}

VAT and SAT linked increased risks for metabolic abnormality in men no matter in the BMI $224 \mathrm{~kg} / \mathrm{m}^{2}$ or in $\mathrm{BMI} \geq 24 \mathrm{~kg} / \mathrm{m}^{2}$, but in women, SAT only increased the risk of MA in BMl $\geq 24 \mathrm{~kg} / \mathrm{m}^{2}$. Deposit of abdominal adipose tissue associated with the metabolic abnormalities. VAT improved the predictive ability of MA.

\section{Introduction}

Obesity, especially central obesity, is a strong risk factor for a variety diseases, such as dyslipidemia, type 2 diabetes, cardiovascular diseases, and all-cause mortality $(1,2)$. Body mass index (BMI) and waist circumference (WC) are two commonly used indicators of obesity. However, BMI by itself does not fully 
characterize adiposity, it is limited by differences in body adiposity for a given BMI across age, gender and ethnicity(3). Asians are generally more likely to have metabolic abnormalities with a lower BMI than Caucasians(4). Although WC reflected central obesity and readily available, do not adequately reflect actual body fat tissue distribution and thus fail to distinguish between abdominal subcutaneous adipose tissue (SAT) and visceral adipose tissue (VAT), and SAT and VAT have different metabolic consequences(5). While reports on the effects of SAT on metabolic abnormality are still inconclusive and even contradictive $(1,6-8)$. Some studies have found that SAT is a beneficial fat depot for type 2 diabetes and metabolic syndrome $(8,9)$, however, other studies have not found a significant correlation between SAT and some metabolic abnormal components $(6,7,10)$. In addition, because of sex-difference in fat depot accumulation between the sexes, they have different effects on metabolic abnormalities. The conclusion that VAT increased the risk of metabolic abnormalities is consistent, but the extent to which it affects different genders is unclear. Some studies have found that higher VAT has a causal relationship with cardiometabolic risk factors, with a larger effect on women(11). While other studies indicated that VAT contributes to a more absolute risk for metabolic factors in men than women(12), highlighting the gender difference in the contribution of regional fat distribution.

A possible reason for these sophisticated associations may due to the distinct fat distribution across ethnic groups $(13,14)$. In addition, the way of measurement of SAT and VAT, and the adjustment of confounding factors were also likely reasons. Currently, accurate assessment of regional fat depots requires imaging with radiographic techniques such as computed tomography (CT) and magnetic resonance imaging (MRI)(15). MRI-based adipose tissue measurements that directly quantify abdominal fat compartments, such as VAT and SAT. In the Chinese population, limited studies had probed into the effect of MRI-measured SAT, VAT on metabolic disorders in different sex. Therefore, we explored the relationship between SAT, VAT and metabolic abnormalities in men and women, respectively.

\section{Materials And Methods}

\section{Subjects}

The subjects were recruited from our previous baseline investigation of metabolic syndrome investigation in China in 2010. The detailed information was previously described(16). In this study, a subpopulation from two communities in Hangzhou $(n=1170)$ and Chengdu $(n=761)$ was included. Subjects were excluded if they had (1) severe chronic diseases including cardiovascular diseases, cancers, kidney dysfunction and other chronic wasting diseases, (2) missing data on anthropometric information, SAT and VAT, a total of 1388 eligible subjects were included at last.

This study was approved by the institutional review board at Zhejiang University, Zhejiang, China. All participants were given their written informed consents.

\section{SAT and VAT measurements}


Abdominal adipose tissue was measured by nuclear magnetic resonance imaging (MRI) using a wholebody imaging system (SMT-100, Shimadzu Co, Japan) with TR-500 and TE-200 of SE. MRI scan was performed at the level of umbilicus between L4 and L5 with the subject in the supine position. The areas of subcutaneous and visceral abdominal adipose tissue were calculated using sliceOmatic software (version 4.2).

\section{Covariant assessment}

With a standard questionnaire, the information including age, gender, smoking (current, former, and never), alcohol drinking behaviors (never, moderate, and heavy), and menstrual history (for women) were collected. Current smoking was defined as smoking at least one cigarette per day and lasting for one year. Former smoking was considered as quitting smoking for at least one year. According to the frequency of drinking, they were classified as heavy drinking, moderate drinking and never: more than three times per week was categorized as heavy drinking. Anthropometric variables were collected by trained investigators with a standard protocol, including weight, height, WC, systolic blood pressure, and diastolic blood pressure. Body mass index (BMI) was calculated as weight (in kilograms) divided by the square of height (in meters). Waist circumference (WC) was measured at the midpoint between the iliac crest and lowest rib. Blood pressure was surveyed in a sitting position with a mercury sphygmomanometer. Systolic blood pressure (SBP) and diastolic blood pressure (DBP) were measured as the average of three repeat measurements with at least 30-s intervals.

The overnight fasting blood samples were collected for each subject. Total triglycerides (TG), total cholesterol (TC), high-density lipoprotein cholesterol (HDL-C), low-density lipoprotein cholesterol (LDL-C) were measured by biochemical auto-analyzers (Hitachi 7060, Tokyo, Japan). Fasting plasma glucose (FPG) was analyzed with a glucose oxidase method with the Beckman Glucose Analyzer (Beckman Instruments, Irvine, CA, USA).

The abnormal metabolic components were defined by the metabolic syndrome definition of the International Diabetes Federation (IDF) criteria in 2005, including elevated TG $\geq 1.7 \mathrm{mmol} / \mathrm{L}$, low HDL$\mathrm{C}<1.03 \mathrm{mmol} / \mathrm{L}$ (in men), $<1.29 \mathrm{mmol} / \mathrm{L}$ (in women); elevated $\mathrm{FPG} \geq 5.6 \mathrm{mmol} / \mathrm{L}$ or have a history of diabetes, or using drugs of anti-diabetes; elevated SBP $>130 \mathrm{mmHg}$, or $\mathrm{DBP}<85 \mathrm{mmHg}$ or using antihypertensive drug therapy. Metabolic abnormality (MA) was defined as more than one abnormally metabolic components, metabolic normality $(\mathrm{MN})$ was defined as zero or only one metabolic component with abnormality(16).

\section{Statistical analysis}

Continuous variable was presented as mean \pm standard deviation or median (inter-quartiles range). Categorical variable was shown as number (\%). Student's t-test or the Wilcoxon rank-sum test was used to compare continuous variables. Chi-square test was used to compare categorical variables. The subjects were divided into four groups by quartiles of SAT and VAT, respectively, with the first quartile (Q1) as the reference group. The ORs and $95 \% \mathrm{Cls}$ for each quartile by sex were calculated using multiple 
logistic regression, adjusted for age, BMI (for overall), smoke, drink, menstrual history (for women). Considering different distributions between men and women, further analysis was performed by stratifying sex. The significant level was set at $P<0.05$. The main two packages of "Predict ABEL" and "pROC" were used to calculate the net reclassification improvement (NRI), integrated discrimination improvement (IDI), area under curve (AUC) and so on. The software IBM SPSS Statistics version 25.0 and $\mathrm{R}$ 3.6.3 were used to analyze the data.

\section{Results}

\section{The baseline characteristics of subjects}

Table 1

Characteristics of the subjects stratified by sex

\begin{tabular}{|c|c|c|c|c|}
\hline Characteristics & Total $(n=1388)$ & Men $(n=622)$ & Women $(n=766)$ & $P$ \\
\hline Age(years) & $53.8 \pm 7.1$ & $53.6 \pm 7.1$ & $53.9 \pm 7.1$ & 0.539 \\
\hline BMI $\left(\mathrm{kg} / \mathrm{m}^{2}\right)$ & $23.70 \pm 2.99$ & $24.18 \pm 2.99$ & $23.31 \pm 2.93$ & $<0.001$ \\
\hline WC (cm) & $79.3 \pm 9.0$ & $83.3 \pm 8.4$ & $76.0 \pm 8.1$ & $<0.001$ \\
\hline WHR & $0.87 \pm 0.07$ & $0.90 \pm 0.06$ & $0.84 \pm 0.06$ & $<0.001$ \\
\hline SAT area $\left(\mathrm{cm}^{2}\right)$ & $148.5(112.9-194.7)$ & $123.2(98.1-149.8)$ & 178.2(139.1-221.6) & $<0.001$ \\
\hline VAT area $\left(\mathrm{cm}^{2}\right)$ & $69.5(45.5-107.2)$ & $91.0(55.1-127.4)$ & $60.4(43.3-79.6)$ & $<0.001$ \\
\hline $\mathrm{SBP}(\mathrm{mmHg})$ & $120.5 \pm 15.8$ & $123.7 \pm 15.4$ & $117.8 \pm 15.6$ & $<0.001$ \\
\hline $\mathrm{DBP}(\mathrm{mmHg})$ & $79.5 \pm 9.8$ & $82.4 \pm 9.8$ & $77.2 \pm 9.2$ & $<0.001$ \\
\hline FPG (mmol/L) & $5.12 \pm 1.17$ & $5.23 \pm 1.41$ & $5.03 \pm 0.92$ & 0.003 \\
\hline OGTT-2h (mmol/L) & $6.65 \pm 3.30$ & $6.97 \pm 3.93$ & $6.38 \pm 2.65$ & 0.002 \\
\hline $\mathrm{TC}(\mathrm{mmol} / \mathrm{L})$ & $5.28 \pm 1.08$ & $5.13 \pm 1.00$ & $5.40 \pm 1.13$ & $<0.001$ \\
\hline $\mathrm{TG}(\mathrm{mmol} / \mathrm{L})$ & $1.30(0.90-1.87)$ & $1.47(1.00-2.19)$ & $1.20(0.85-1.70)$ & $<0.001$ \\
\hline $\mathrm{HDL}-\mathrm{C}(\mathrm{mmol} / \mathrm{L})$ & $1.48 \pm 0.36$ & $1.36 \pm 0.33$ & $1.58 \pm 0.36$ & $<0.001$ \\
\hline LDL-C(mmol/L) & $2.59 \pm 0.67$ & $2.57 \pm 0.67$ & $2.61 \pm 0.66$ & 0.273 \\
\hline $\mathrm{MA}(\mathrm{n}, \%)$ & $667(48.1 \%)$ & $357(57.4)$ & $310(40.5)$ & $<0.001$ \\
\hline \multicolumn{5}{|c|}{$\begin{array}{l}\text { Data are presented as means } \pm \text { standard deviation or medians (inter-quartile ranges) or } n \\
\text { (percentage). BMI, body mass index, WC, waist circumference; WHR, waist-to-hip ratio; SAT, } \\
\text { subcutaneous adipose tissue; VAT, visceral adipose tissue; SBP, systolic blood pressure; DBP, diastolic } \\
\text { blood pressure; FPG, fasting plasma glucose; OGTT-2h, } 2 \text { hour post oral glucose tolerance test; TC, } \\
\text { total cholesterol; TG, triglyceride; HDL-C, high density lipoprotein cholesterol, LDL-C, low density } \\
\text { lipoprotein cholesterol; MA: Metabolic abnormality, which was defined as metabolic abnormal } \\
\text { components } \geq 2 \text {, which were based on the definition of metabolic syndrome (IDF 2005). }\end{array}$} \\
\hline
\end{tabular}


The baseline characteristics by sex are summarized in Table1. In the 1388 subjects, 622 (44.8\%) were men, $766(55.2 \%)$ were women. The mean age was 53.8 years old $(S D=7.1)$. Men has higher VAT (91.0, $95 \% \mathrm{Cl}=55.1-127.4)$ than women $(60.4,95 \% \mathrm{Cl}=43.3-79.6)(\mathrm{P}<0.05)$, and lower levels of SAT (123.2, $95 \% \mathrm{Cl}=98.1-149.8)$ than women $(178.2,95 \% \mathrm{Cl}=139.1-221.6)(P<0.05)$. Compared with women, men were more likely to have higher BMI, WC, hip circumference (HC), WHR, VAT, SBP, DBP, FPG, OGTT-2h, TG, and a higher prevalence of metabolic abnormality; women had higher TC and HDL-C (all the Pvalues < 0.05).

\section{Levels of SAT, VAT in different metabolic status stratified by sex and BMI}

Table 2 presents the levels of SAT, VAT in metabolic abnormality stratified by sex and BMI. In Men, subjects with MA had significant higher levels of SAT $(130.6,95 \% \mathrm{Cl}=106.2-159.5)$ and VAT $(110.5,95 \%$ $\mathrm{Cl}=75.2-136.8$ ) than $\mathrm{MN}$ group (SAT: $110.8,95 \% \mathrm{Cl}=80.3-141.3$; VAT: $64.5,95 \% \mathrm{Cl}=32.1-101.2$ ) (all the $P$ values $<0.05)$. Similar results were found in women (Table2).

Table 2

Levels of SAT, VAT in different metabolic status stratified by sex and BMI

Men

MN

$110.8(80.3-$

141.3)

64.5(32.1-

101.2)
$130.6(106.2-$

159.5)

$110.5(75.2-$

136.8)

Women

$\begin{array}{llll}P & \mathrm{MN} & \mathrm{MA} & P\end{array}$

$\begin{array}{llll}P & \mathrm{MN} & \mathrm{MA} & P\end{array}$

$\begin{array}{llll}P & \mathrm{MN} & \mathrm{MA} & P\end{array}$

Overall

VAT

BMI $24 \mathrm{~kg} / \mathrm{m}^{2}$

SAT

90.7(66.92-

112.2)

106.5(82.7-

121.4)

0.001

154.5(125.9-

183.1)

170.3(133.9-

205.9)

191.0(148.8-

239.3)

0.001

53.4(36.0-

68.8)

75.2(55.9-

107.2)

0.001

48.9(34.2-

62.6)

77.10(53.90-

104.05)

<.001

62.6)

$42.1(22$.
$67.8)$

0.001

$\mathrm{BMI} \geq$

$24 \mathrm{~kg} / \mathrm{m}^{2}$

SAT

139.5(116.1-

161.7)

148.0(125.6-

180.9)

0.004

126.6(101.7-

155.8)

97.8(69.4-

122.9)

$<$

0.001

$6.4(47.0$

92.7)

212.5(180.8-

250.6)

66.4(47.0-

Data are presented as medians (inter-quartile ranges). BMI, body mass index.MN: Metabolic normality, which was defined as abnormally metabolic components $\leq 1$; MA: Metabolic abnormality, which was defined as metabolic abnormal components $\geq 2$. 
When stratified by levels of BMI at $24 \mathrm{~kg} / \mathrm{m}^{2}$, similar results were also found for SAT and VAT in men and only VAT in women irrespectively of BMI levels. In women, borderline significance was found for SAT in $\mathrm{BMI} \geq 24 \mathrm{~kg} / \mathrm{m}^{2}(P=0.066)$ and no significance in $\mathrm{BMl}<24 \mathrm{~kg} / \mathrm{m}^{2}(P>0.05)$.

\section{The associations of different levels of SAT, VAT with metabolic abnormality stratified by sex and BMI}

Table 3 shows the associations of SAT, VAT with metabolic abnormality stratified by sex and BMI after adjusted for age, BMI (for overall), smoke, drink, and menstrual history (for women). In men and women, levels of VAT were significantly correlated with higher risks for MA ( $p$ for trend $<0.05$ ). Comparing with the reference group of the first quartile (Q1), OR in fourth quartile (Q4) was $6.537(95 \% \mathrm{Cl}=3.394-12.591)$ in men and $3.364(95 \% \mathrm{Cl}=1.898-5.962)$ in women, however, no significance was found in SAT. 
Table 3

The relationships between SAT, VAT and metabolic abnormality stratified by sex and BMI

Men

n $\quad \% \quad$ OR $(95 \% \mathrm{Cl})$
Women

n $\quad \% \quad$ OR $(95 \% \mathrm{Cl})$

Overall

SAT

Q1

60

38.7 ref

$\begin{array}{lll}60 & 31.4 & \text { ref }\end{array}$

Q2

91

$58.7 \quad 1.458(0.878-2.421)$

$70 \quad 36.6 \quad 0.833(0.518-1.338)$

Q3

$97 \quad 62.6 \quad 1.344(0.762-2.371)$

$76 \quad 39.4 \quad 0.667(0.403-1.103)$

Q4

$109 \quad 70.3 \quad 1.391(0.707-2.735)$

$10454.5 \quad 0.576(0.319-1.040)$

$P$ for trend

0.445

0.05

VAT

Q1

$45 \quad 29$ ref

$\begin{array}{lll}40 & 20.9 & \text { ref }\end{array}$

Q2

85

Q3

$105 \quad 67.3 \quad 3.939(2.199-7.053)$

$122 \quad 78.7$

6.537(3.394-

12.591)

$P$ for trend

$<0.001$

$<0.001$

BMI $₫ 24 \mathrm{~kg} / \mathrm{m}^{2}$

SAT

Q1

$\begin{array}{lll}50 & 38.8 \quad \text { ref }\end{array}$

$\begin{array}{lll}47 & 30.1 & \text { ref }\end{array}$

Q2

\section{5}

Q3 and Q4

32

56.8

2.062(1.177-3.613)

$49 \quad 30.8 \quad 0.911(0.549-1.511)$

Q4

$P$ for trend

0.009

$31 \quad 29.5 \quad 0.896(0.510-1.575)$

$20 \quad 47.6 \quad 1.631(0.781-3.407)$

$56.1 \quad 2.121(1.103-4.078)$

0.463

VAT

Q1

36

28.1 ref

$29 \quad 19.5 \quad$ ref

Q2

52

Q3 and Q4

$48 \quad 71.6$

3.505(1.945-6.314)

$46 \quad 31.1 \quad 1.631(0.936-2.845)$

$40 \quad 33.6 \quad 1.770(0.988-3.168)$

11.795) 


\section{Men}

Q4

$P$ for trend

$<0.001$

Women

$32 \quad 69.6$

7.422(3.422-

16.095)

$<0.001$

$\mathrm{BMI} \geq 24 \mathrm{~kg} / \mathrm{m}^{2}$

SAT

\begin{tabular}{lllllll} 
Q1 & 10 & 38.5 & ref & 13 & 37.1 & ref \\
\hline Q2 & 37 & 61.7 & $2.516(0.949-6.672)$ & 21 & 65.6 & $\begin{array}{l}4.753(1.531- \\
\end{array}$ \\
& & & & & $14.755)$
\end{tabular}

Q3 $\quad \begin{array}{llllll}70 & 64.8 & \mathbf{2 . 8 2 3}(\mathbf{1 . 1 3 2}-7.039) & 45 & 51.1 & 2.474(0.968-6.323)\end{array}$

Q4 $\quad \begin{array}{llllll}104 & 71.7 & \mathbf{3 . 8 6 2 ( 1 . 5 7 3 - 9 . 4 8 4 )} & 84 & 56.4 & \mathbf{2 . 5 0 2 ( 1 . 0 2 1 - 6 . 1 2 9 )}\end{array}$

$P$ for trend

0.005

0.35

VAT

\begin{tabular}{|c|c|c|c|c|c|c|}
\hline Q1 & 9 & 33.3 & ref & 11 & 26.2 & ref \\
\hline Q2 & 33 & 48.5 & $1.703(0.656-4.420)$ & 18 & 40.9 & $2.185(0.803-5.944)$ \\
\hline Q3 & 71 & 64 & $3.244(1.305-8.064)$ & 37 & 50.7 & $2.576(1.024-6.478)$ \\
\hline Q4 & 108 & 81.2 & $\begin{array}{l}\text { 7.836(3.086- } \\
19.893)\end{array}$ & 97 & 66.9 & $\begin{array}{l}4.607(1.909- \\
11.118)\end{array}$ \\
\hline
\end{tabular}

$P$ for trend

$<0.001$

$<0.001$

Data are presented as medians (inter-quartile ranges) or OR $(95 \% \mathrm{Cl})$. BMI, body mass index. The ORs was adjusted for age, BMI (for overall), smoke, drink, and menstrual history (for women). Men: SAT

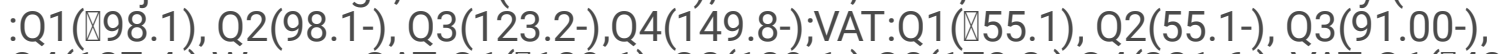
Q4(127.4-);Women:SAT:Q1(『139.1), Q2(139.1-),Q3(178.2-),Q4(221.6-); VAT:Q1(『43.0), Q2(43.0-), Q3(60.4-), Q4(79.6-).

When stratified by levels of BMI at $24 \mathrm{~kg} / \mathrm{m}^{2}$, significant associations were found for VAT in men and women irrespectively of BMI levels. However, for SAT, different effects were found between men and women. In men, no matter in the BMI $24 \mathrm{~kg} / \mathrm{m}^{2}$ or in BMI $\geq 24 \mathrm{~kg} / \mathrm{m}^{2}$, levels of SAT were consistently associated with the risk of MA ( $p$ for trend囚0.05). However, in women, significantly increased risk was only found in BMI $\geq 24 \mathrm{~kg} / \mathrm{m}^{2}$. Supplemental table 1 and 2(See Additional File 1) showed the relationship between SAT, VAT and metabolic components, indicating that SAT may be a protective factor for highFPG in women, and the OR for Q4 was 0.383(0.185-0.792) ( $p$ for trend 80.05 ).

\section{The Predictive Ability Of Vat On Metabolic Abnormality}


Table 4 describes the predictive ability of VAT on metabolic abnormality. In men, the AUC of VAT was 0.727, which was significantly higher than that of $\mathrm{BMI}(0.658,95 \% \mathrm{Cl}=0.614-0.701)$ and $\mathrm{WC}(0.688$, $95 \% \mathrm{Cl}=0.646-0.730$ ) (all the $\mathrm{P}$ values $<0.05$ ). When compared with BMI or WC, VAT improved the predictive value of MA, NRI $(95 \% \mathrm{Cl})$ were $0.139(0.070,0.208)$ and $0.106(0.038,0.173)$, respectively; IDI $(95 \% \mathrm{Cl})$ were $0.074(0.053-0.095)$ and $0.046(0.026-0.066)$, respectively.

Table 4

The predictive values on metabolic abnormality in BMI- WC- SAT- and VAT

\begin{tabular}{|c|c|c|c|c|c|c|c|}
\hline & AUC $(95 \% \mathrm{Cl})$ & $Z$ & $P$ & $\operatorname{NRI}(95 \% \mathrm{Cl})$ * & $P$ & IDI $(95 \% \mathrm{Cl})$ * & $P$ \\
\hline \multicolumn{8}{|l|}{ men } \\
\hline BMI & $\begin{array}{l}0.658(0.614- \\
0.701)\end{array}$ & & & & & & \\
\hline WC & $\begin{array}{l}0.688(0.646- \\
0.730)\end{array}$ & & & & & & \\
\hline VAT & $\begin{array}{l}0.727(0.687- \\
0.767)\end{array}$ & -3.864 & $\varangle 0.001$ & $\begin{array}{l}0.139(0.070- \\
0.208)\end{array}$ & 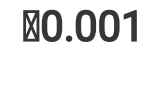 & $\begin{array}{l}0.074(0.053- \\
0.095)\end{array}$ & 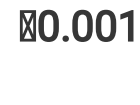 \\
\hline VAT* & & -2.458 & 0.014 & $\begin{array}{l}0.106(0.038- \\
0.173)\end{array}$ & 0.003 & $\begin{array}{l}0.046(0.026- \\
0.066)\end{array}$ & $\llbracket 0.001$ \\
\hline \multicolumn{8}{|c|}{ women } \\
\hline BMI & $\begin{array}{l}0.666(0.627- \\
0.705)\end{array}$ & & & & & & \\
\hline WC & $\begin{array}{l}0.693(0.655- \\
0.732)\end{array}$ & & & & & & \\
\hline VAT & $\begin{array}{l}0.712(0.674- \\
0.749)\end{array}$ & -2.562 & 0.01 & $\begin{array}{l}0.112(0.037- \\
0.188)\end{array}$ & 0.004 & $\begin{array}{l}0.050(0.028- \\
0.072)\end{array}$ & $\llbracket 0.001$ \\
\hline VAT* & & -1.115 & 0.265 & $\begin{array}{l}0.042(-0.031- \\
0.114)\end{array}$ & 0.261 & $\begin{array}{l}0.028(0.007- \\
0.049)\end{array}$ & 0.008 \\
\hline
\end{tabular}

Similar results were found in women(Table4), the AUC of VAT was 0.712 , which was significantly higher than that of $\mathrm{BMI}(0.666,95 \% \mathrm{Cl}=0.627-0.705)$ and $\mathrm{WC}(0.693,95 \% \mathrm{Cl}=0.655-0.732)$ (all the $\mathrm{P}$ values < 0.05). Compared with BMI and WC, VAT also improved the predictive value.

\section{Discussion}

In this cross-sectional study, we found that higher VAT rather than SAT increased risk for MA in men, and for women, the correlation of SAT only existed in BMI $\geq 24 \mathrm{~kg} / \mathrm{m}^{2}$. Higher VAT showed a significant 
positive association with MA in men and women, independent of BMI. VAT improved the predictive ability of MA comparing with BMI and WC. Deposit of abdominal adipose tissue associated with the risk of MA.

There are some differences between SAT and VAT in anatomical, cellular, molecular, physiological, clinical and so on(17). VAT has been considered the more pathogenic adipose tissue compartment compared with SAT(18). This may be related to the biological function of VAT, VAT was a metabolically active organ, which included more non-adipocytes including macrophages, immune cells, preadipocytes and fibroblasts, and could secrete amounts of inflammation mediators to induce metabolism impairments $(17,19-21)$. In addition, the high lipolytic activity of VAT and its accompanied inflammation response also contribute to abnormal lipogenesis, glucose homeostasis, and vascular health $(22,23)$. Therefore, it is plausible that higher VAT increased the risk for the metabolic abnormalities. With regards to the contribution of VAT in different sex, inconclusive results were reported(11, 12, 24-26). Several Caucasian population studies showed the associations of VAT with type 2 diabetes, hypertension and hyperlipidemia were stronger in women $(11,24,27)$. In our supplemental table3(See Additional File 1), we observed that the effect of VAT on high TG and low-HDL were higher in men, indicating that VAT may have more striking effect on the lipid metabolism in men than women. Another possible reason was that only limited confounding factors were adjusted, which may have affected the results. Extending studies of the Chinese population needed to be expanded to determine gender differences in the contribution of VAT.

SAT is known to have adverse effects on a variety of metabolic risk factors and may have unique pathogenic properties independent of BMI $(1,7,24,28)$, and the effects of different levels of SAT on cardiometabolic factors are inconsistent $(1,7,14,18,24,29)$. In line with previous studies(29-31), our study (See Supplementary table 1 and 2, Additional File 1) showed higher SAT did not link to increased risk for hypertension, higher TG, and lower HDL-C independent of BMI, and SAT may be a protective factor for blood glucose. Several studies for European or African population found that SAT had independent associations with high blood pressure and $\operatorname{HDL}-\mathrm{C}(1,15,25)$, suggesting the distinct effect of SAT across ethnics. A possible explanation for this gender difference in SAT was the distinct sex steroid hormone profiles since these sex hormones are important factors in regulating the adipose tissue distribution and energy metabolism $(32,33)$. For the protective effect of SAT, there were also several hypotheses to explain this observation. One is with smaller adipocyte, SAT was more insulin sensitive, and have a larger capacity to uptake fatty acids and triglycerides, thus can act as a powerful buffer to prevent excess fat into non-adipose tissue(34). On the other hand, SAT could secrete more favorable adipokines such as adiponectin, which had anti-diabetes and antiatherogenic properties $(17,22)$. Therefore, the different effects of SAT on metabolic outcomes may be related to its biological function.

Previous studies have shown that baseline and changes in VAT were independent predictors of future dyslipidemia, but BMI and SAT were not associated with future development of atherosclerotic dyslipidemia(35). The outcome was consistent to our study that VAT was a better indicator for predicting MA when compared to BMI and WC. Finally, VAT was more strongly associated with MA than BMI, WC, and SAT. 
There are some advantages in our study. Areas of SAT and VAT were measured using MRI, which was the gold standard method of determining abdominal adipose tissue. The data, including anthropometric and questionnaire-based information, were collected by trained health professionals, and the biochemical measurements followed the standard protocols. Our study also has some limitations. First, we cannot infer causality between the fat indices and the metabolic abnormality because of the cross-sectional design. Second, limited confounding factors were included in this study, for example, regional fat distribution, such as deep SAT and superficial SAT, and drug use were not included, which may bias the results. Thirdly, the sample size of this study was relatively small. Finally, our data were based on only one single ethnic group, thus the results may not be applied to other ethnicities.

\section{Conclusions}

In brief, deposit of abdominal adipose tissue associated with the risk of MA. VAT positively increased risks for metabolic abnormality in both of men and women, different effects were found between men and women. In women, SAT only increased the risk of MA in BMI $\geq 24 \mathrm{~kg} / \mathrm{m}^{2}$; VAT increased the predictive ability of on MA comparing with BMI and WC. Distribution of abdominal fat associates with its biological effects.

\section{Abbreviations}

SAT, subcutaneous adipose tissue;

VAT, visceral adipose tissue;

MRI, nuclear magnetic resonance imaging;

BMI, body mass index;

WC, waist circumference;

WHR, waist-to-hip ratio;

SBP, systolic blood pressure;

DBP, diastolic blood pressure;

FPG, fasting plasma glucose;

OGTT-2h, 2 hour post oral glucose tolerance test;

TC, total cholesterol;

TG, triglyceride; 
HDL-C, high density lipoprotein cholesterol;

LDL-C, low density lipoprotein cholesterol;

MA, metabolic abnormality;

MN: metabolic normality.

\section{Declarations}

\section{Acknowledgement}

We also would like to thank all the participants and investigators that took part in this study.

\section{Authors' contributions}

Conceptualization, YZ; Data curation, QC; Formal analysis, QC and XS; Funding acquisition, $X Z$ and $Y Z$; Investigation, QL and JZ; Project administration, $X Z$ and $Y Z$; Supervision, $Y Z$; Writing - original draft, $X Z$ and QC; Writing - review \& editing, YZ, QW and ZC. All authors read and approved the final manuscript.

\section{Funding}

This work was supported by the grants from National Key Research and Development Program of China (2017YFC0907004), Hangzhou Science and Technology Project (20171226Y27), and Zhejiang Health Science and technology Project (2021KY268). The funder has no role in the design of the study, collection, analysis, and interpretation of data.

\section{Availability of data and materials}

The datasets used and/or analyzed during the current study are available from the corresponding author on reasonable request.

\section{Ethics approval and consent to participate}

The study was approved by the institutional review board at Zhejiang University, Zhejiang, China. All participants were given their written informed consents.

\section{Consent for Publication}

Not applicable.

\section{Competing interests}

The authors declare that they have no competing interests. 
1. Xuhui Zhang. Email: 994028847@qq.com.

2. Qiannan Chen. Email: 2716068678@qq.com.

3. Xiaohui Sun. Email: 20191030@zcmu.edu.cn.

4. Qiong Wu. Email: qiong1012@zju.edu.cn.

5. Zongxue Cheng. Email: 21918694@zju.edu.cn.

Correspondence: Yimin Zhu. Telephone: 0086-571-88208194, Email: zhuym@zju.edu.cn;

Jiaqiang Zhou. Email: zhoujq27@yahoo.com;

Qingguo Lv. Email: lqg3713@163.com.

\section{References}

1. Abraham TM, Pedley A, Massaro JM, Hoffmann U, Fox CS. Association between visceral and subcutaneous adipose depots and incident cardiovascular disease risk factors. Circulation. 2015;132(17):1639-47.

2. Rothney MP, Catapano AL, Xia J, Wacker WK, Tidone C, Grigore L, et al. Abdominal visceral fat measurement using dual-energy X-ray: association with cardiometabolic risk factors. Obesity (Silver Spring Md). 2013;21(9):1798-802.

3. Jackson AS, Stanforth PR, Gagnon J, Rankinen T, Leon AS, Rao DC, et al. The effect of sex, age and race on estimating percentage body fat from body mass index: The Heritage Family Study. International journal of obesity related metabolic disorders: journal of the International Association for the Study of Obesity. 2002;26(6):789-96.

4. Appropriate body-mass. index for Asian populations and its implications for policy and intervention strategies. Lancet. 2004;363(9403):157-63.

5. Keum N, Lee DH, Kim R, Greenwood DC, Giovannucci EL. Visceral adiposity and colorectal adenomas: dose-response meta-analysis of observational studies. Ann Oncol. 2015;26(6):1101-9.

6. Chen P, Hou X, Hu G, Wei L, Jiao L, Wang H, et al. Abdominal subcutaneous adipose tissue: a favorable adipose depot for diabetes? Cardiovascular diabetology. 2018;17(1):93.

7. Liu J, Fox CS, Hickson DA, May WD, Hairston KG, Carr JJ, et al. Impact of abdominal visceral and subcutaneous adipose tissue on cardiometabolic risk factors: the Jackson Heart Study. J Clin Endocrinol Metab. 2010;95(12):5419-26.

8. Kwon H, Kim D, Kim JS. Body Fat Distribution and the Risk of Incident Metabolic Syndrome: A Longitudinal Cohort Study. Sci Rep. 2017;7(1):10955.

9. Porter SA, Massaro JM, Hoffmann U, Vasan RS, O'Donnel CJ, Fox CS. Abdominal subcutaneous adipose tissue: a protective fat depot? Diabetes Care. 2009;32(6):1068-75.

10. Hoyer D, Boyko EJ, McNeely MJ, Leonetti DL, Kahn SE, Fujimoto WY. Subcutaneous thigh fat area is unrelated to risk of type 2 diabetes in a prospective study of Japanese Americans. Diabetologia. 
2011;54(11):2795-800.

11. Karlsson T, Rask-Andersen M, Pan G, Höglund J, Wadelius C, Ek WE, et al. Contribution of genetics to visceral adiposity and its relation to cardiovascular and metabolic disease. Nat Med. 2019;25(9):1390-5.

12. Chen Y, Zhang Z, Wang J, Sun H, Zhao X, Cheng X, et al. Sex differences in the association of abdominal adipose tissue and anthropometric data with untreated hypertension in a Chinese population. Biology of sex differences. 2020;11(1):38.

13. Lesser IA, Gasevic D, Lear SA. The effect of body fat distribution on ethnic differences in cardiometabolic risk factors of Chinese and Europeans. Appl Physiol Nutr Metab. 2013;38(7):701-6.

14. Rønn PF, Andersen GS, Lauritzen T, Christensen DL, AadahI M, Carstensen B, et al. Ethnic differences in anthropometric measures and abdominal fat distribution: a cross-sectional pooled study in Inuit, Africans and Europeans. J Epidemiol Commun Health. 2017;71(6):536-43.

15. Bertoli S, Leone A, Vignati L, Spadafranca A, Bedogni G, Vanzulli A, et al. Metabolic correlates of subcutaneous and visceral abdominal fat measured by ultrasonography: a comparison with waist circumference. Nutr J. 2016;15:2.

16. Zheng R, Yang M, Bao Y, Li H, Shan Z, Zhang B, et al. Prevalence and Determinants of Metabolic Health in Subjects with Obesity in Chinese Population. Int J Environ Res Public Health. 2015;12(11):13662-77.

17. Ibrahim MM. Subcutaneous and visceral adipose tissue: structural and functional differences. Obes Rev. 2010;11(1):11-8.

18. Tang L, Zhang F, Tong N. The association of visceral adipose tissue and subcutaneous adipose tissue with metabolic risk factors in a large population of Chinese adults. Clin Endocrinol (Oxf). 2016;85(1):46-53.

19. Kahn CR, Wang G, Lee KY. Altered adipose tissue and adipocyte function in the pathogenesis of metabolic syndrome. J Clin Investig. 2019;129(10):3990-4000.

20. Despres JP, Lemieux I, Bergeron J, Pibarot P, Mathieu P, Larose E, et al. Abdominal obesity and the metabolic syndrome: contribution to global cardiometabolic risk. Arterioscler Thromb Vasc Biol. 2008;28(6):1039-49.

21. Misra A, Vikram NK. Clinical and pathophysiological consequences of abdominal adiposity and abdominal adipose tissue depots. Nutrition (Burbank, Los Angeles County. Calif). 2003;19(5):45766.

22. Chait A, den Hartigh LJ. Adipose Tissue Distribution, Inflammation and Its Metabolic Consequences, Including Diabetes and Cardiovascular Disease. Frontiers in cardiovascular medicine. 2020;7:22.

23. Després JP, Lemieux I. Abdominal obesity and metabolic syndrome. Nature. 2006;444(7121):881-7.

24. Fox CS, Massaro JM, Hoffmann U, Pou KM, Maurovich-Horvat P, Liu CY, et al. Abdominal visceral and subcutaneous adipose tissue compartments: association with metabolic risk factors in the Framingham Heart Study. Circulation. 2007;116(1):39-48. 
25. Rønn PF, Andersen GS, Lauritzen T, Christensen DL, Aadahl M, Carstensen B, et al. Abdominal visceral and subcutaneous adipose tissue and associations with cardiometabolic risk in Inuit, Africans and Europeans: a cross-sectional study. BMJ open. 2020;10(9):e038071.

26. Bidulescu A, Liu J, Hickson DA, Hairston KG, Fox ER, Arnett DK, et al. Gender differences in the association of visceral and subcutaneous adiposity with adiponectin in African Americans: the Jackson Heart Study. BMC Cardiovasc Disord. 2013;13:9.

27. Borel AL, Nazare JA, Smith J, Aschner P, Barter P, Van Gaal L, et al. Visceral, subcutaneous abdominal adiposity and liver fat content distribution in normal glucose tolerance, impaired fasting glucose and/or impaired glucose tolerance. Int J Obes (Lond). 2015;39(3):495-501.

28. Oka R, Miura K, Sakurai M, Nakamura K, Yagi K, Miyamoto S, et al. Impacts of visceral adipose tissue and subcutaneous adipose tissue on metabolic risk factors in middle-aged Japanese. Obesity (Silver Spring Md). 2010;18(1):153-60.

29. Matsha TE, Ismail S, Speelman A, Hon GM, Davids S, Erasmus RT, et al. Visceral and subcutaneous adipose tissue association with metabolic syndrome and its components in a South African population. Clin Nutr ESPEN. 2019;32:76-81.

30. Koh H, Hayashi T, Sato KK, Harita N, Maeda I, Nishizawa Y, et al. Visceral adiposity, not abdominal subcutaneous fat area, is associated with high blood pressure in Japanese men: the Ohtori study. Hypertens Res. 2011;34(5):565-72.

31. Zhao X, Gang X, Liu Y, Sun C, Han Q, Wang G. Using Metabolomic Profiles as Biomarkers for Insulin Resistance in Childhood Obesity: A Systematic Review. J Diabetes Res. 2016;2016:8160545.

32. Palmer BF, Clegg DJ. The sexual dimorphism of obesity. Molecular cellular endocrinology. 2015;402:113-9.

33. Karastergiou K. The Interplay Between Sex, Ethnicity, and Adipose Tissue Characteristics. Curr Obes Rep. 2015;4(2):269-78.

34. Kim S, Cho B, Lee H, Choi K, Hwang SS, Kim D, et al. Distribution of abdominal visceral and subcutaneous adipose tissue and metabolic syndrome in a Korean population. Diabetes Care. 2011;34(2):504-6.

35. Hwang YC, Fujimoto WY, Hayashi T, Kahn SE, Leonetti DL, Boyko EJ. Increased Visceral Adipose Tissue Is an Independent Predictor for Future Development of Atherogenic Dyslipidemia. J Clin Endocrinol Metab. 2016;101(2):678-85.

\section{Supplementary Files}

This is a list of supplementary files associated with this preprint. Click to download.

- Additionalfile1.docx 